\title{
Quarkonium spectral functions with complex potential
}

\author{
Chuan Miao, ${ }^{1,2}$ Ágnes Mócsy, ${ }^{3}$ and Péter Petreczky ${ }^{1}$ \\ ${ }^{1}$ Physics Department, Brookhaven National Laboratory, Upton NY 11973 USA \\ ${ }^{2}$ Institute of Nuclear Physics, Johannes Gutenberg-Universität Mainz, \\ Johann-Joachim-Becher-Weg 45, D-55099 Mainz, Germany \\ ${ }^{3}$ Pratt Institute, Department of Math and Science, Brooklyn, NY 11205, USA
}

(Dated: November 7, 2018)

\begin{abstract}
We study quarkonium spectral functions at high temperatures using potential model with complex potential. The real part of the potential is constrained by the lattice QCD data on static quark anti-quark correlation functions, while the imaginary part of the potential is taken from perturbative calculations. We find that the imaginary part of the potential has significant effect on quarkonium spectral functions, in particular, it leas to the dissolution of the $1 \mathrm{~S}$ charmonium and excited bottomonium states at temperatures about $250 \mathrm{MeV}$ and melting of the ground state bottomonium at temperatures slightly above $450 \mathrm{MeV}$.
\end{abstract}




\section{INTRODUCTION}

It is known from lattice QCD that strongly interacting matter undergoes a transition to a deconfined phase also called quark gluon plasma (QGP) characterized by chiral symmetry restoration and color screening [1]. Quarkonia have been suggested as experimental signature of deconfinement by Matsui and Satz [2]. Namely, it has been argued that color screening in a deconfined QCD medium will suppress the existence of quarkonium states, signaling the formation of QGP in heavy-ion collisions. Although this idea was proposed a long time ago, attempts to calculate quarkonium spectral functions from first principle QCD have been made only relatively recently. The idea behind these attempts is to calculate the correlation function of the corresponding meson operators in Euclidean time in lattice QCD and to reconstruct the quarkonium spectral functions using the Maximum Entropy Method (MEM) [3-9]. It turned out that the spectral functions of S-wave quarkonium in the deconfined phase are similar to those in the confined phase, and this fact was interpreted as survival of the ground state quarkonium in the high temperature region. However, as this was pointed out in Ref. [8] details of the spectral functions cannot be resolved at high temperatures due to limited extent of the Euclidean time direction (see Ref. [10] for recent review on this issue). Furthermore, lattice artifacts at small separations (large momenta) further complicate the extraction of the spectral functions 11]. On the other hand, the temperature dependence of the quarkonium correlation functions can be studied reliably in lattice QCD. It was found that in the pseudo-scalar and vector channels corresponding to ground state quarkonia the temperature dependence of quarkonium correlation functions is either very small or moderate [6, 8, 9]. At the same time large temperature dependence in the scalar and axialvector channels was observed. These findings were interpreted as survival of the ground state quarkonium and the dissolution of the excited P-states, which fitted well into the expected sequential melting pattern. However, a detailed study of the temperature dependence of the quarkonium correlators revealed that almost the entire temperature dependence of the quarkonium correlators is due to the zero modes not to the dissolution of the bound states [12], thus challenging the above interpretation.

With progress in lattice calculations of the correlation functions of static quark antiquark pairs and better understanding of the color screening phenomenon there has been a renewed interest in potential models at finite temperature [13 19]. The basic idea of this approach is to assume that medium effects on quarkonium properties can be incorporated in a temperature dependent potential and use lattice QCD to constrain its form. It is not clear whether and under which circumstances medium effects can be characterized by a temperature dependent potential. Fortunately, the effective field theory approach to heavy quark bound states provides an answer [20]. Heavy quark bound states at zero temperature are characterized by three distinct energy scales $m \gg m v \gg m v^{2}$ corresponding to the heavy quark mass, the typical momenta inside the bound state, and the typical binding energy respectively. The heavy quark velocity $v$ can be treated as a small expansion parameter and furthermore, in the weak coupling regime $v \sim \alpha_{s}$. This allows construction of sequence of the effective field theories called NRQCD and pNRQCD by integrating out the scales $m$ and $m v$ [21] (see Ref. [22] for a detailed review on this subject). The degrees of freedom in pNRQCD are the singlet and the octet meson fields composed of the heavy quark and antiquark and interacting with ultra-soft gluons, i.e. gluons on energy scale $m v^{2}$. The singlet and octet potentials appear as the parameters of the effective field theory Lagrangian and therefore can be defined at any order in perturbation theory or even non-perturbatively 
[21]. The effective field theory approach can be generalized to non-zero temperature, but the presence of additional thermal scales $T, m_{D} \sim g T$ and $g^{2} T$ ( with $g$ being the gauge coupling, $g^{2}=4 \pi \alpha_{s}$ ) make the analysis more complicated [20]. In the case when the binding energy is smaller than the temperature scales the potential receives thermal corrections which have both real and imaginary parts and one gets different versions of thermal pNRQCD. The precise form of the real and imaginary parts of the thermal corrections depends on the relation of the scales $1 / r \sim m v, T$ and $g T[20]{ }^{1}$

In the past quarkonium spectral functions in QGP have been calculated in Refs. 15, 17 19] using potential model with real potentials. The aim of the present paper is to perform a calculation of quarkonium spectral functions in the deconfined phase using a potential model inspired by thermal pNRQCD, i.e. using a complex potential. The rest of this paper is organized as follows: In section II we review what is known about the potential at finite temperature from lattice QCD and perturbation theory. In section III we present our numerical results for the quarkonium spectral functions. In section IV we discuss the temperature dependence of the corresponding Euclidean time quarkonium correlation functions. Finally, section $\mathrm{V}$ contains our conclusions.

\section{THE POTENTIAL AT FINITE TEMPERATURE}
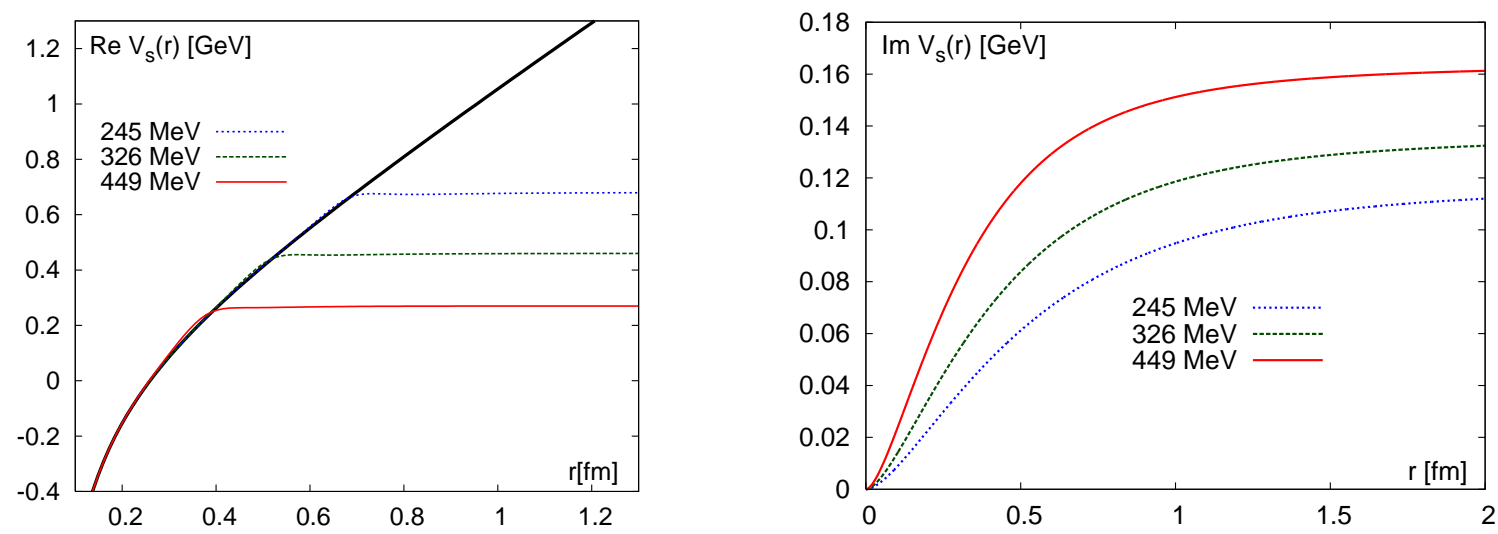

FIG. 1: The real (left) and the imaginary part (right) of the potential used in our analysis.

We are interested in studying quarkonium spectral functions in the temperature region where the medium significantly changes the properties of the bound states, i.e. the bound states are close to being dissolved. This means that the binding energies are significantly reduced from their vacuum values and eventually vanish at some temperature called the point of zero binding. Thus, in this situation the binding energy is the smallest scale in the problem and all the medium effects can be incorporated in the potential. If we start from the pNRQCD and neglect the octet-singlet interactions the dynamics of the singlet quark antiquark field is given by the free field equation which is the form of the Schrodinger equation

${ }^{1}$ The presence of the imaginary part in the finite temperature potential was first pointed out in Ref. [23] by analyzing Wilson loops in the hard thermal loop approximation. In the effective field theory framework it can be shown that this analysis corresponds to calculation of the potential in the distance regime $1 / r \sim g T$. 
(e.g. see discussions in Ref. [22]). Then the calculation of the correlation function of singlet fields in real time or equivalently the quarkonium spectral function in non-relativistic limit is reduced to calculating the Green function of the Schrödinger equation [24]

$$
\begin{gathered}
{\left[-\frac{1}{m} \vec{\nabla}^{2}+V(r, T)-E\right] G^{n r}\left(\vec{r}, \vec{r}^{\prime}, E, T\right)=\delta^{3}\left(r-r^{\prime}\right)} \\
\sigma(\omega, T)=\left.\frac{6}{\pi} \operatorname{Im} G^{n r}\left(\vec{r}, \overrightarrow{r^{\prime}}, E\right)\right|_{\vec{r}=\vec{r}^{\prime}=0} \quad E=\omega-2 m .
\end{gathered}
$$

The potential entering the above equation is complex [20, 23]. Its form is quite complicated even in the weakly coupled regime as the form of the thermal corrections depends on the relation of the scales $1 / r, T, m_{D} \sim g T$. Furthermore, in the interesting temperature regime the separation of the above scales does not hold and the potential is effected by the nonperturbative scales $g^{2} T, \Lambda_{Q C D}$. Therefore we have to rely on the lattice calculations to constrain the form of the potential.

The interaction of a static quark and anti-quark $(Q \bar{Q})$ at finite temperature is usually studied on the lattice in terms of the so-called singlet correlation functions $G_{1}(r, T)=$ $\operatorname{Tr}\left\langle W(r) W^{\dagger}(0)\right\rangle$ with $W$ being the temporal Wilson line. It has been calculated in pure gauge theory [25, 26], in 3-flavor QCD [28], in 2-flavor QCD [27] and more recently also in $2+1$ flavor QCD [29 31]. Since the expression for $G_{1}(r, T)$ is not gauge invariant the calculations are performed in Coulomb gauge. The singlet correlator can be viewed as the correlation function of color singlet static meson field at Euclidean time separation $\tau=1 / T$. Therefore it is related to the static energy of a $Q \bar{Q}$ pair at finite temperature (see discussion in Ref. [10]), though this relation can be quite complicated. The other type of static quark anti-quark correlation function that is calculated on the lattice is the Polyakov loop correlator $G(r, T)=\left\langle\operatorname{Tr} W(r) \operatorname{Tr} W^{\dagger}(0)\right\rangle$ which is gauge invariant. Unfortunately, its temperature dependence is much more complicated, because it is sensitive to the excited states $[29]^{2}$. We also note the properties of the singlet correlator that are discussed below are not specific to the Coulomb gauge. In fact, the singlet correlation function defined through periodic Wilson loops at finite temperature shares all the properties of the Coulomb gauge singlet correlator [36].

The logarithm of the singlet correlator defines the so-called singlet free energy $F_{1}(r, T) / T=-\ln G_{1}(r, T)$. For the further discussion it is useful to briefly discuss the properties of the singlet free energy. At short distances the singlet free energy coincides with the zero temperature potential. In the deconfined region, medium effects become significant at distances $r_{o}=0.4 \mathrm{fm} /\left(T / T_{\text {dec }}\right)$ with $T_{\text {dec }}$ being the deconfinement temperature [25]. At distances $r_{e}=r T \geq 0.8$ the singlet free energy is exponentially screened, i.e. $F_{1}(r, T)-F_{\infty}(T)$ falls off exponentially [29]. The constant $F_{\infty}(T)$ is twice the free energy of an isolated static quark and it is related to the gauge invariant renormalized Polyakov loop expectation value $L_{r e n}(T)=\exp \left(-F_{\infty}(T) /(2 T)\right)$. We expect that the real part of the potential $\operatorname{Re} V(r, T)$

\footnotetext{
${ }^{2}$ In leading order of perturbation theory the Polyakov loop correlator can be written as the thermal average of color singlet and octet contribution [32, 33]. Recently this has been proved at next-to-leading order in Ref. 34], where it was also shown how to define rigorously the singlet and octet contribution using pNRQCD. In the low temperature limit it can be shown that all excited states contribute to the Polyakov loop correlator with the same weight as the ground state [35], while for the singlet correlator the contribution of the excited states is suppressed [36].
} 
entering in Eq. (2.1) will share the same qualitative features as the singlet free energy, in particular medium effects will be significant at some distance $r_{o} \leq r_{\text {med }}(T) \leq r_{e}$. Its value will depend on the choice of $r_{\text {med }}(T)$. In our analysis we choose $r_{\text {med }}(T)=r_{e}$ and assume that for distances smaller than $r_{\text {med }}$ the real part of the potential coincides with the zero temperature potential, while for $r>r_{m e d}(T)$ it is exponentially screened ${ }^{3}$. Furthermore, we use a smooth interpolation between these two regimes as described in Ref. [19]. This procedure also determines the asymptotic value of the potential $V_{\infty}(T)$ which turns out to be close to value of the internal energy of static $Q \bar{Q}$ pair at infinite separation $U_{\infty}(T)$ [19]. In Fig. 1 we show the real part of the potential for several values of the temperature Since on general grounds we expect $F_{\infty} \leq V_{\infty}(T) \leq U_{\infty}$ the above choice of the potential provides the most binding potential compatible with the lattice data. Following Ref. [19] we call this choice of $\operatorname{Re} V(r, T)$ the maximally binding potential (in Ref. [19] it was also labeled as set II). Unfortunately lattice QCD cannot yet determine the imaginary part of the potential. Therefore here we have to rely on perturbation theory. However, even in perturbation theory the form of the imaginary part is only know for certain limiting cases corresponding to some hierarchy of the relevant scales $1 / r, T, m_{D}$ [20]. Therefore for the imaginary part we choose the perturbative hard thermal loop (HTL) result obtained in Ref. [23]

$$
\operatorname{Im} V^{H T L}(r, T)=-\frac{i g^{2} T C_{F}}{4 \pi} \phi\left(m_{D} r\right), \phi(x)=2 \int_{0}^{\infty} \frac{d z z}{\left(z^{2}+1\right)^{2}}\left[1-\frac{\sin (z x)}{z x}\right],
$$

which is in principle valid only for distances $r>1 / m_{D}$ [20]. However, the above expression has the nice feature that it vanishes for distances which are much smaller than the inverse temperature. For the numerical values of $g^{2}(T)$ and $m_{D}(T)$ entering the above formulas following Ref. [24] we choose

$$
g^{2}(T)=\frac{8 \pi^{2}}{9 \ln \left(9.082 T / \Lambda_{\overline{M S}}\right)}, \quad m_{D}^{2}(T)=\frac{8 \pi^{2}}{3 \ln \left(7.547 T / \Lambda_{\overline{M S}}\right)}, \quad \Lambda_{\overline{M S}}=300 \mathrm{MeV} .
$$

Due to the large numerical pref-actor in the argument of the logarithm the imaginary part of the potential is not too large even at temperatures $T \simeq 200 \mathrm{MeV}$. Therefore we may consider this choice as a conservative lower bound for it. The resulting $\operatorname{Im} V(r, T)$ is shown in Fig. 1. In the next section we present charmonium and bottomonium spectral functions for this choice of the potential which is maximally binding and minimally dissipative.

\section{NUMERICAL RESULTS FOR THE SPECTRAL FUNCTIONS}

With the potential discussed in the previous section we have solved the Schrödinger equation for the non-relativistic Green functions and thus calculated the spectral function for S-wave quarkonium. We used the numerical algorithm described in Ref. [24]. First for a reference we need to calculate the zero temperature spectral function. At zero temperature the potential has no imaginary part, however, Eq. (1) cannot be solved numerically if the imaginary part of the potential is strictly zero. Therefore we need to introduce a small imaginary part to the potential when solving the Schrödinger equation. In the past we used a constant imaginary part equal to $0.01 \mathrm{~m}$ for charmonium and $0.003 \mathrm{~m}$ for bottomonium

3 Note that the nature of this screening is strongly non-perturbative [37, 38]. 

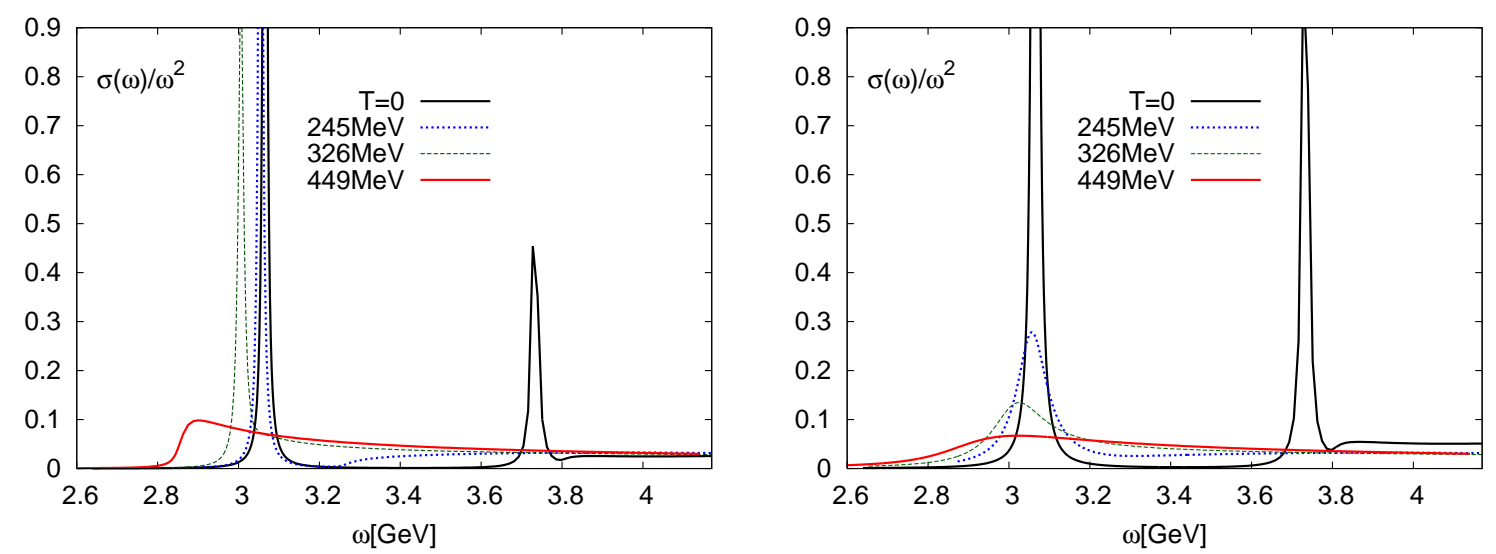

FIG. 2: The charmonium spectral functions function calculated in potential model neglecting the imaginary part of the potential (left) and using both the real and imaginary part of the potential (right). The maximally binding potential has been used for the real part.

[18, 19]. Here we use $\operatorname{Im} V^{H T L}(r, T=148 \mathrm{MeV}) / 10$ for the imaginary part. It is better than the previous choice as it gives less artificial width for the ground state. Furthermore, we would like to incorporate the physics of the string breaking into the zero temperature spectral functions. Following Ref. [19] we do this by replacing the Coulomb plus linear form of the potential by a Yukawa form at $r>1.1 \mathrm{fm}$. The parameters of the zero temperature potential can be found in [19]. For charm and bottom quark mass we choose $m_{c}=1.29 \mathrm{GeV}$ and $m_{b}=4.67 \mathrm{GeV}$ respectively. This gives a reasonably good description of the quarkonium spectrum at zero temperature as shown in Table I. The $T=0$ spectral functions obtained

\begin{tabular}{|c|c|c||c|c|c|}
\hline \multicolumn{3}{|c|}{ Charmonia } & \multicolumn{3}{c|}{ Bottomonia } \\
\hline State & Model & PDG & State & Model & PDG/LQCD \\
\hline $1 S$ & 3.067 & 3.068 & $1 S$ & 9.451 & 9.443 \\
$2 S$ & 3.720 & 3.674 & $2 S$ & 9.988 & 10.016 \\
$1 P$ & 3.536 & 3.525 & $3 S$ & 10.357 & 10.355 \\
& & & $1 P$ & 9.864 & 9.900 \\
& & & $2 P$ & 10.247 & 10.260 \\
\hline
\end{tabular}

TABLE I: Spin-averaged charmonium and bottomonium masses in $\mathrm{GeV}$ at zero temperature calculated in our model and compared to the experimental values [39]. To obtain the $2 \mathrm{~S}$ spin averaged bottomonium mass we used the lattice QCD value of $\eta_{b}(2 S)$ mass from Ref. [40].

using the above procedure are shown in Fig. 2 and Fig. 3 for charmonium and bottomonium respectively.

We would like to understand the effect of color screening, encoded in the real part of the potential, as well as the effects of dissipation, encoded in the imaginary part of the potential on the quarkonium spectral functions. Therefore we first calculated the quarkonium spectral functions neglecting the imaginary part of the potential. In terms of the numerics this means that we used $\operatorname{Im} V^{H T L}(r, T) / 10$ in our calculations. The corresponding charmonium and bottomonium spectral functions are shown in the left panels of Fig. 2 and Fig. 3 respectively. We see clear peak like structures in the spectral functions which persist to temperature of 
$326 \mathrm{MeV}$ for charmonium and $449 \mathrm{MeV}$ for bottomonium. In the case of bottomonium we also see a remnant of the $2 S$ state in the spectral function at $T=245 \mathrm{MeV}$. This is consistent with the results obtained in potential models, in particular, with the calculation of Ref. [17] that used internal energy as a potential.

The situation changes dramatically when the imaginary part of the potential $\operatorname{Im} V(r, T)=$ $\operatorname{Im} V^{H T L}(r, T)$ is taken into account. The corresponding spectral functions are also shown in the right panels of Fig. 2 and Fig. 3 for charmonium and bottomonium respectively. In the charmonium spectral function we see a very broad peak at $T=245 \mathrm{MeV}$, which disappears at higher temperatures. In the bottomonium spectral function the $2 \mathrm{~S}$ peak disappears slightly above $T=245 \mathrm{MeV}$, while the ground state peak becomes very broad at $T=450 \mathrm{MeV}$. These findings for the spectral functions are consistent with upper bounds on the quarkonium dissociation temperatures obtained in Ref. [19] by comparing the temperature dependence of the binding energies and the thermal widths, since the lattice data used in both studies correspond to a deconfinement temperature of $T_{d e c} \simeq T_{c} \simeq 204 \mathrm{MeV}^{4}$.

We note that the choice of the real part of the potential used here gives the largest possible binding energies. Nevertheless, the binding energies of all quarkonium states, except for 1S bottomonium are smaller than the temperature for $T \geq 245 \mathrm{MeV}$ and drop rapidly with increasing temperature [19]. Thus, our approach of putting all medium effects into the potential should be at least marginally correct. Binding energies are significantly smaller for other choices of the potential. In particular, a theoretically motivated choice of the potential which is close to the free energy was considered in Ref. [19], where it was called set I. For this potential we see melting of charmonium ground state at $T \simeq 250 \mathrm{MeV}$ even when no imaginary part is considered. At the same time the bottomonium spectral functions are not very different compared to the ones calculated with the maximally binding potential. It is interesting to see how much our results change when switching the potential to set I and keeping the imaginary part of the potential. The corresponding numerical data are shown in Fig. 4. As one can see from the figure changing the real part of the potential has little effect on the bottomonium spectral functions when the imaginary part is present. The dissolution of the excited states and the broadening of the ground state peak are mostly determined by the imaginary part. This also means that the microscopic mechanism behind the melting of charmonium and bottomonium states could be quite different. For ground state charmonium screening effect play and important role, while for the ground state bottomonium the melting will occur due to the large imaginary part of the potential at high temperatures.

\section{EUCLIDEAN TIME CORRELATION FUNCTIONS}

From the calculated quarkonium spectral functions it is easy to calculate the corresponding Euclidean time correlation functions

$$
G(\tau, T)=\int_{0}^{\infty} d \omega \sigma(\omega, T) K(\tau, \omega, T), K\left((\tau, \omega, T)=\frac{\cosh (\omega(\tau-1 /(2 T)))}{\sinh (\omega /(2 T))} .\right.
$$

It is important to compare the temperature dependence of the correlation functions obtained in potential model with the results of the lattice QCD calculations. The temperature dependence of the correlation function on the lattice is studied in terms of the ratio

\footnotetext{
${ }^{4}$ In Ref. [19] the dissociation temperatures were given in units of $T_{c}$.
} 

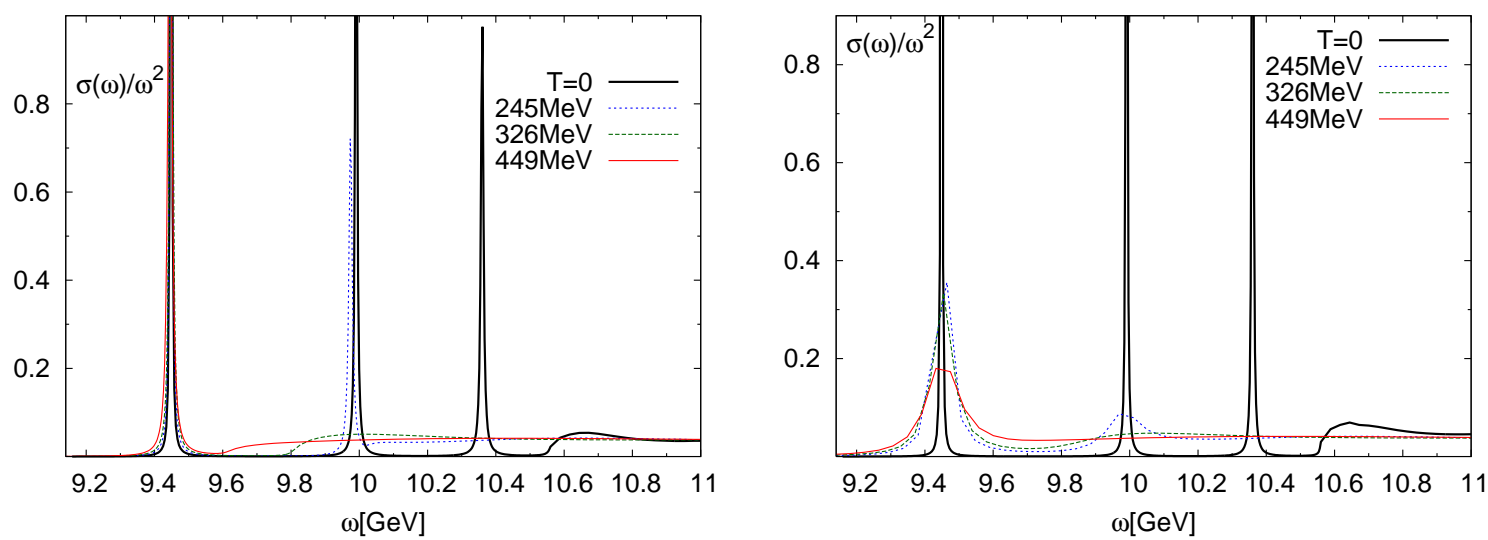

FIG. 3: The bottomonium spectral functions function calculated in potential model neglecting the imaginary part of the potential (left) and using both the real and imaginary part of the potential (right). The maximally binding potential has been used for the real part.

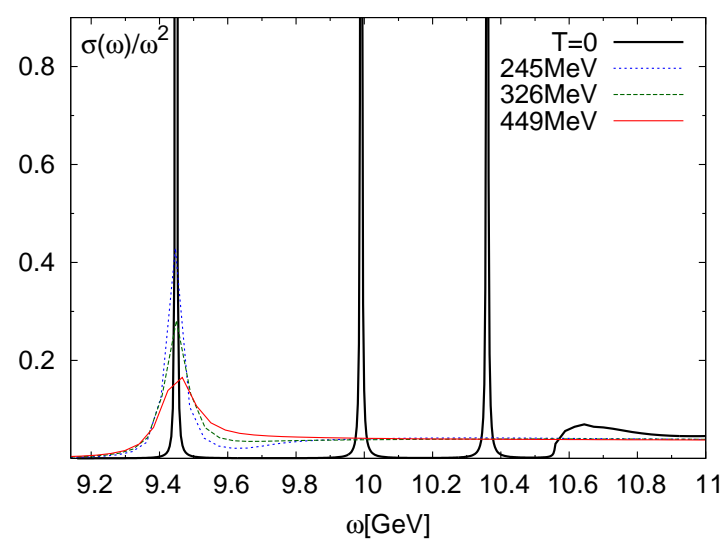

FIG. 4: The bottomonium spectral functions function calculated using the complex potential with real part corresponding to set I of Ref. [19].

$G(\tau, T) / G_{r e c}(\tau, T)$, where

$$
G_{r e c}(\tau, T)=\int_{0}^{\infty} d \omega \sigma(\omega, T=0) K(\tau, \omega, T) .
$$

In the lattice calculations this ratio for pseudo-scalar channel that corresponds to S-wave quarkonia, was found to be close to one [6 -8, 12]. Potential model calculation with real potential could reproduce this feature. Therefore it is interesting to see how close is $G(\tau, T) / G_{r e c}(\tau, T)$ to unity when the imaginary part of the potential is taken into account. The spectral functions calculated in the potential model are not reliable far away from the threshold because of relativistic effects. Therefore in Refs. [18, 19] the spectral functions calculated in the potential model were smoothly matched to the perturbative result for the spectral functions at some higher energy and the ratios $G(\tau, T) / G_{r e c}(\tau, T)$ have been calculated from these matched spectral functions. In this study we did not follow this procedure and only used the spectral functions calculated in the potential model when constructing $G /(\tau, T) / G_{r e c}(\tau, T)$. Our results for the ratio of the correlators is shown in Fig. 5. In the case of the charmonium this ratio stays close to unity even when the complex potential is used. However, $G(\tau, T) / G_{r e c}(\tau, T)$ is noticeable smaller compared to the calculations where the 

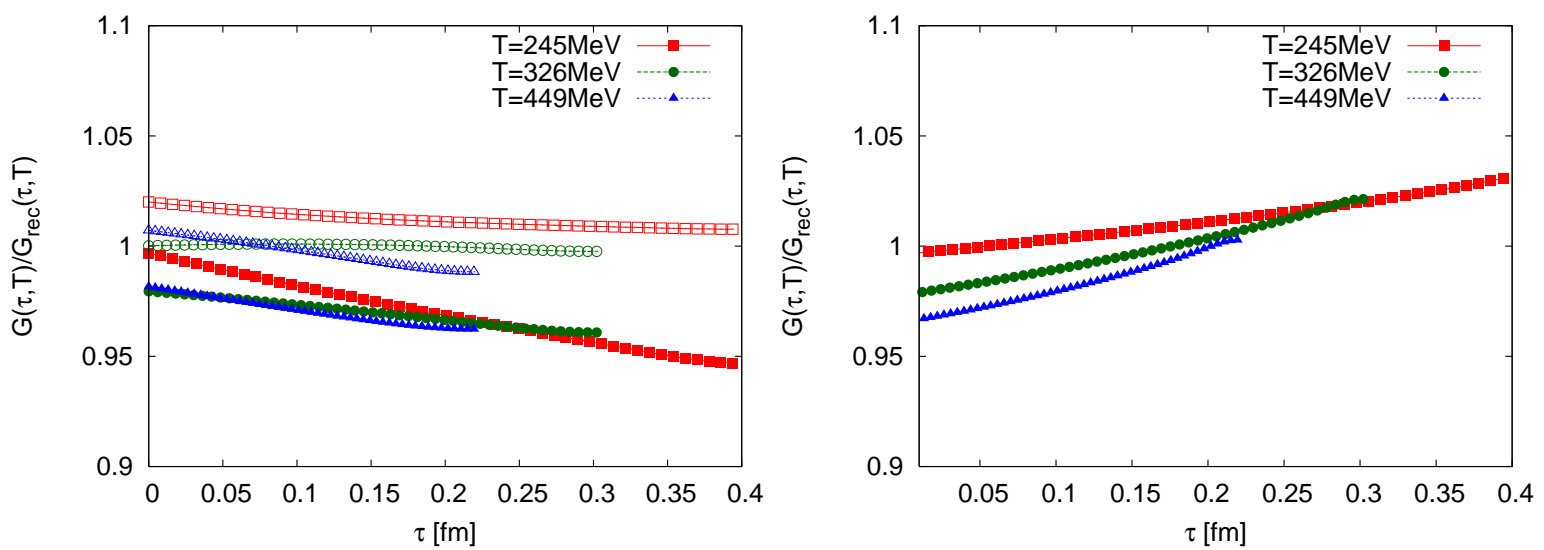

FIG. 5: The ratio $G(\tau, T) / G_{r e c}(\tau, T)$ of charmonium (left) and bottomonium (right) correlators for different temperatures calculated with complex potential. Open symbols in the left plot correspond to the calculation where the imaginary part of the potential was neglected.

imaginary part is neglected shown as open symbols in the figure. In the bottomonium case the imaginary part of the potential had a significant effect and the ratio $G(\tau, T) / G_{r e c}(\tau, T)$ is no longer close to unity in odds with the available lattice data [7, 8]. It is possible that this is due to fact that time scale relevant for the ground state bottomonium is smaller than the time scales related to the medium and modeling of the medium effects by a $T$-dependent potential is not accurate enough. However, the in-medium potential based description should work better for the excited bottomonium states due to their smaller binding energies. Therefore we constructed the ratio $G(\tau, T) / G_{r e c}(\tau, T)$ assuming that the ground state bottomonoium is unmodified and using only the spectral function above $\omega=9.7 \mathrm{GeV}$ in the calculation of the correlation functions. The corresponding results are also shown in Fig. 5. As one can see from the figure the in-medium changes of the bottomonium spectral functions above $9.7 \mathrm{GeV}$, in particular, the meting of excited bottomonium states do not produce any large change in the Euclidean correlation functions. The problem of bottomonium spectral functions, however, needs further investigations in light of recent attempts to calculate the properties of ground state bottomonium at high temperatures using perturbative QCD [41] and new lattice calculations bottomonium correlators in NRQCD at finite temperature [42]. We also note that since we do not match the spectral functions to the perturbative form at higher energies the calculated $G(\tau, T) / G_{r e c}(\tau, T)$ shows larger deviation from unity, especially at shorter distance compared to previous studies [18, 19].

\section{CONCLUSIONS}

In summary, we have calculated the quarkonium spectral functions in the deconfined region using pNRQCD inspired potential model with complex potential. For the real part of the potential we considered the lattice QCD motivated maximum binding potential first introduced in Ref. [19], while for the imaginary part we took the perturbative estimate from [23]. We find in particular that $J / \psi$ peak disappear for temperatures above $245 \mathrm{MeV}$ and $\Upsilon(1 S)$ peak disappears for temperatures above $450 \mathrm{MeV}$. We found that dissipative effects encoded in the imaginary part of the potential play a crucial role in the quarkonioum spectral functions and dissolution of quarkonium states. Similar conclusion have been made very recently in the analysis of charmonium spectral functions using the T-matrix approach, 
where dissipative effects are encoded in the heavy quark self energy [43]. This study also finds no bound state peaks in QGP. In the future it would be interesting to study other choices for the imaginary part of the potential, which could lower the dissociation temperature for the ground state bottomonium as well as to study the corresponding Euclidean time correlation functions more in detail.

\section{Acknowledgements}

This work has been supported by contract DE-AC02-98CH10886 with the U.S. Department of Energy. The numerical calculations of the quarkonium spectral functions has been performed and the algorithm and the code described in Ref. [24]. We thank Mikko Laine for providing this code for us.

[1] P. Petreczky, Nucl. Phys. Proc. Suppl. 140 (2005) 78-91; Mod. Phys. Lett. A25 (2010) 3081-3092; J. Phys. Conf. Ser. 16 (2005) 169-173

[2] T. Matsui and H. Satz, Phys. Lett. B 178 (1986) 416

[3] T. Umeda, K. Nomura and H. Matsufuru, Eur. Phys. J. C 39S1 (2005) 9

[4] M. Asakawa and T. Hatsuda, Phys. Rev. Lett. 92 (2004) 012001

[5] F. Karsch et al., Nucl. Phys. A715 (2003) 701-704 [hep-ph/0209028].

[6] S. Datta et al., Phys. Rev. D 69, 094507 (2004); J. Phys. G G30 (2004) S1347-S1350

[7] S. Datta, et al., AIP Conf. Proc. 842 (2006) 35-37

[8] A. Jakovác, P. Petreczky, K. Petrov and A. Velytsky, Phys. Rev. D 75 (2007) 014506

[9] G. Aarts et al., Phys. Rev. D 76 (2007) 094513

[10] A. Bazavov, P. Petreczky, A. Velytsky, [arXiv:0904.1748 [hep-ph]].

[11] F. Karsch, et al., Phys. Rev. D68 (2003) 014504; S. Stickan, et al., Nucl. Phys. Proc. Suppl. 129 (2004) 599-601; I. Wetzorke et al., Nucl. Phys. Proc. Suppl. 106 (2002) 510-512

[12] P. Petreczky, Eur. Phys. J. C62 (2009) 85-93

[13] S. Digal, P. Petreczky and H. Satz, Phys. Rev. D 64 (2001) 094015; Phys. Lett. B514, 57-62 (2001)

[14] C. Y. Wong, Phys. Rev. C 72 (2005) 034906

[15] Á. Mócsy and P. Petreczky, Phys. Rev. D 73 (2006) 074007; Eur. Phys. J. C 43 (2005) 77

[16] W. M. Alberico, A. Beraudo, A. De Pace and A. Molinari, Phys. Rev. D 75 (2007) 074009

[17] D. Cabrera and R. Rapp, Phys. Rev. D 76 (2007) 114506

[18] Á. Mócsy and P. Petreczky, Phys. Rev. D 77 (2008) 014501

[19] Á. Mócsy and P. Petreczky, Phys. Rev. Lett. 99 (2007) 211602

[20] N. Brambilla, et al., Phys. Rev. D78 (2008) 014017

[21] N. Brambilla, et al., Nucl. Phys. B566 (2000) 275

[22] N. Brambilla, et al., Rev. Mod. Phys. 77 (2005) 1423; N. Brambilla, S. Eidelman, B. K. Heltsley et al., [arXiv:1010.5827 [hep-ph]]; N. Brambilla et al. [ Quarkonium Working Group Collaboration ], [hep-ph/0412158]

[23] M. Laine, et al., JHEP 0703, 054 (2007)

[24] Y. Burnier, M. Laine, M. Vepsalainen, JHEP 0801 (2008) 043 
[25] O. Kaczmarek, et al., Phys. Lett. B543 (2002) 41-47; [hep-lat/0207002]. Int. J. Mod. Phys. A20 (2005) 3789-3792.

[26] S. Digal, S. Fortunato, P. Petreczky, Phys. Rev. D68 (2003) 034008 [hep-lat/0304017].

[27] O. Kaczmarek, F. Zantow, Phys. Rev. D71, 114510 (2005)

[28] P. Petreczky, K. Petrov, Phys. Rev. D70 (2004) 054503

[29] P. Petreczky, J. Phys. G 37 (2010) 094009

[30] O. Kaczmarek, PoS CPOD07 (2007) 043

[31] K. Petrov [ RBC-Bielefeld Collaboration ], PoS LAT2007 (2007) 217

[32] L. D. McLerran, B. Svetitsky, Phys. Rev. D24 (1981) 450

[33] S. Nadkarni, Phys. Rev. D33 (1986) 3738

[34] N. Brambilla, J. Ghiglieri, P. Petreczky et al., Phys. Rev. D82 (2010) 074019

[35] O. Jahn, O. Philipsen, Phys. Rev. D70 (2004) 074504

[36] A. Bazavov, P. Petreczky, A. Velytsky, Phys. Rev. D78 (2008) 114026; PoS LATTICE2008 (2008) 186

[37] K. Kajantie, et al., Phys. Rev. Lett. 79 (1997) 3130-3133; M. Laine, O. Philipsen, Nucl. Phys. B523 (1998) 267-289; Phys. Lett. B459 (1999) 259-264

[38] F. Karsch, M. Oevers and P. Petreczky, Phys. Lett. B442 (1998) 291-299; [hep-lat/9807035].

F. Karsch and P. Petreczky, Nucl. Phys. Proc. Suppl. 83 (2000) 393-395

[39] K. Nakamura et al. (Particle Data Group), J. Phys. G 37 (2010) 075021

[40] A. Gray et al., Phys. Rev. D 72 (2005) 094507

[41] N. Brambilla, et al., JHEP 1009 (2010) 038 [arXiv:1007.4156 [hep-ph]].

[42] G. Aarts, S. Kim, M. P. Lombardo et al., [arXiv:1010.3725 [hep-lat]].

[43] F. Riek, R. Rapp, [arXiv:1012.0019 [nucl-th]]. 